artelogie

\section{Artelogie}

Recherche sur les arts, le patrimoine et la littérature de l'Amérique latine

$10 \mid 2017$

Après le paysage : l'art, l'inscription et la représentation de la nature en Amérique latine aujourd'hui

\title{
Les paysages du sud : paysages critiques et dystopie
}

Biennale de San Paulo, 2016

Maria José de Azevedo Marcondes

\section{OpenEdition \\ Journals}

\section{Édition électronique}

URL : http://journals.openedition.org/artelogie/885

DOI : $10.4000 /$ artelogie. 885

ISSN : 2115-6395

Éditeur

Association ESCAL

Référence électronique

Maria José de Azevedo Marcondes, «Les paysages du sud : paysages critiques et dystopie »,

Artelogie [En ligne], 10 | 2017, mis en ligne le 05 avril 2017, consulté le 20 avril 2019. URL : http:// journals.openedition.org/artelogie/885; DOI : 10.4000/artelogie.885

Ce document a été généré automatiquement le 20 avril 2019.

Association ESCAL 


\section{Les paysages du sud : paysages critiques et dystopie}

Biennale de San Paulo, 2016

Maria José de Azevedo Marcondes

\section{Introduction}

1 Cet article présente tout d'abord une lecture du projet des commissaires de la 32ème biennale de São Paulo, dans le chapitre «Paysages du Sud: une autre cartographie pour l'art » et cherche à situer la Biennale de São Paulo dans le contexte d'autres projets d' expositions d'art telles que la Documenta de Kassel et la Biennale JOGIA XIV du Nigéria, lesquelles, au moyen du terme "Anthropophagiser le Sud", cherchent une perspective post- colonialiste de la culture, et plus spécifiquement, de l'art contemporain. A partir de ce panorama de la 32ème Biennale est élaborée la réflexion - «Le paysage dans l'œuvre de Carolina Caycedo»: dont l'analyse se tourne vers les déplacements des représentations du paysage dans la contemporanéité. Ces dernières sont très distantes des représentations du paysage comme réflexe de l'expérience directe ou transmises par des artistes dont le regard est européen; thème dont nous débattons d'ailleurs également, dans la perspective théorique du paysage en tant que construction sociale. De cette manière, l'analyse de l'oeuvre Le peuple Xingu, Le peuple Doux veut mettre en evidence le projet de la 32ème Biennale.

2 En suivant la thématique "Une approche des Paysages critiques ou Paysages du Sud", nous présentons la lecture critique de plusieurs expositions d'Art Contemporaine au Brésil et de quelques oeuvres d'artistes visuels qui ont fait des représentations picturales du paysage dans l'art contemporain, en cherchant, par ce biais, à déconstruire un imaginaire teinté par les processus de colonisation européenne du continent latinoaméricain. Les analyses discursives de ces expositions et de ces oeuvres proposent de mettre en évidence le fait que la construction des représentations artistiques rend compte de la pensée autonome sur l'art contemporain dans ce l'on appelle communément le « sud global ». 
3 L'analyse critique de ces expositions et de ces oeuvres se propose de montrer que la construction de répresentations artistiques qui rendent compte d'une pensée autonome sur l'art contemporain, dans ce qui est dénommé "sud global ", est antérieure à cette Biennale de São Paulo ; néanmoins, cet évènement a alimenté et généré un champ de débat plus large.

4 L'œuvre de Carolina Caycedo - artiste visuelle colombienne née à Londres -, intitulée Le peuple Xingu, Le peuple Doux a été produite pour la 32ème Biennale de São Paulo ${ }^{1}$, sur le thème de " $\mathrm{L}^{\prime}$ Incertitude Vivante ». Pour cette exposition, les commissaires ${ }^{2}$ ont fortement mis l'accent sur les questions concernant les savoirs traditionnels ou marginalisés, les effets anthropiques de l'homme sur la nature et les conditions d'instabilité et de déplacement qui caractérisent l'habitat humain. Dans cet article, nous utiliserons les postulats épistémologiques basés sur le concept d' "épistémologie du Sud ». "Épistémologies du Sud» est compris comme la "récupération des savoirs et des pratiques des groupes sociaux qui, par le biais du capitalisme et du colonialisme, ont été historiquement et sociologiquement placés dans la position d'être si seul objet ou la matière première des connaissances dominantes, considérées comme les seuls valables" ${ }^{\text {. Nous soulignons }}$ par ailleurs que l'auteur Boaventura dos Santos a écrit un texte sur le thème central de la 32ème Biennale de São Paulo - L' Incertitude Vivante - dans le catalogue de la Biennale d'art ${ }^{4}$.

5 L'œuvre artistique de Carolina Caycedo se développe dans un cadre social en participant activement au mouvement de l'économie solidaire et de la résistance territoriale dans des pays tels que la Colombie, le Mexique et le Brésil; pour ne citer que ces exemples en Amérique latine. Dans le même temps, l'œuvre de Carolina Caycedo reprend le thème du paysage dans l'art contemporain; sujet sur lequel nous nous concentrerons, particulièrement sur la notion de paysage dans l'art comme une construction sociale ${ }^{5}$.

6 Ces propositions artistiques, selon nous, doivent faire l'objet d'une recherche à la lumière de la pensée philosophique critique et peuvent être comprises et interprétées selon de nombreuses nuances discursives et théoriques de l'art contemporain. Nous signalons, toutefois que nous ne concevons pas le projet des commissaires comme un désir de retour à des structures économiques et socioculturelles d'autres périodes historiques, qui pourrait, selon l'analyse de Clément Rosset, instaurer un " naturalisme conservateur" ${ }^{6} \mathrm{Ce}$ n'est pas non plus une possible interprétation romantique de l'art contemporain ${ }^{7}$, telle que proposée par la philosophe et critique d'art Danielle Cohn dans une réflexion critique sur l'œuvre d'Anselm Kiefer présentée à la Mostra Monumenta (Grand Palais, 2007) et à Barjac (France) ${ }^{8}$.

7 Dans ce texte, nous avons fait dialoguer les œuvres de Caycedo avec les concepts centraux de l'épistémologie du Sud, tels que formulés par Boaventura dos Santos - la sociologie des absences, la sociologie des situations d'urgence, l'écologie de savoirs et la traduction interculturelle - (SANTOS, 2010 : p. 460). Ces concepts pourraient, à notre avis, reconfigurer un large champ d'expériences poétiques avec des pouvoirs différents, mis en place dans la 32ème Biennale de São Paulo, en niant, notamment, toute possibilité de matrices discursives de natures nostalgiques, de formes de vie passées. Carolina Caycedo et Alia Farid, parmi d'autres grands artistes de la 32ème Biennale, exposent des représentations du paysage qui cherchent la décolonisation des connaissances et des pratiques artistiques.

8 La question du thème de la place des artistes visuels devant la contingence écologique, comme les changements climatiques, les catastrophes environnementales et plus 
généralement l'anthropisation de la nature, exige, bien sûr, la compréhension de la notion ou du concept de réalité et sa transformation en une nouvelle réalité, par le biais de poétiques visuelles.

9 Pour une analyse critique de ces processus, nous devons, sans aucun doute, interroger les notions de réel et de réalité et le léger clivage entre le réel et l'art dans la contemporanéité. Dans cette partie de l'analyse, nous utilisons la pensée critique du philosophe américain Arthur Danto (1981) dont l'œuvre écrite il y a trois décennies, offre encore un fondement théorique valable, notamment ses arguments proposés dans « $L a$ Transfiguration du banal: une philosophie d'art ». Cette anthologie de l'œuvre d'art facilite l'analyse de la différence ontologique entre les œuvres d'art et les objets communs, différence qui est posée dans la représentation et dans l'histoire mais qui peut pourtant ne pas exister tant les deux catégories peuvent être indiscernables.

\section{Paysages du sud : une autre cartographie pour l'art}

10 La 32ème Biennale de São Paulo a pris comme point de départ le paradigme de l'incertitude sur "les manières dont nous comprenons ou pas notre être dans le monde aujourd'hui: dégradation de l'environnement, violence, menaces aux communautés et à la diversité culturelle: réchauffement climatique, effondrements économiques et politiques, catastrophes naturelles, vie ravagée par des atrocités, la maladie et la faim sont la matière qui sont autour de nous", selon les termes du commissaire de l'exposition Jochen Voltz (VOLTZ, 2016 :p.21). Mobilisés par des questions soulevées par le terme « Anthropocène » - utilisé par différentes disciplines pour désigner une période historique caracterisée par $l^{\prime}$ intense impact des activités humaines sur les écosystèmes mondiaux ${ }^{9}$, les commissaires ont d'abord choisi le thème et le titre de la Biennale - «Mesures de l'Incertitude » ${ }^{10}$. Ensuite, ils, ils se sont orientés vers une recherche de la "pensée cosmologique, intelligence environnementale et collective et l'écologie systémique et naturel 》 (VOLTZ, 2016: p. 22) et sont arrivés sur le thème " L' Incertitude Vivante ", dans une perspective post-colonialiste de l'art et de la culture et, certainement, influencés par la pensée "perspectiviste amérindienne $»^{11}$.

11 Les commissaires de l'Exposition ont compris le rôle actuel de la Biennale comme celui d'une "plateforme d'articulations entre la pensée critique et l'expérimentation artistique dans une région géographique, indépendamment des frontières nationales ", pour reprendre les mots du commisaire de l'exposition (VOLTZ, 2016 : p. 25). L'expansion de la Biennale au-delà de ses frontières temporelles et territoriales a entraîné un très vif débat sur les savoirs collectifs et les récits évolutionnaires qui ont été débattus lors des activités nommées «Journées d'Étude ». À Santiago du Chili, ce débat a porté sur la cosmologie Rapa Nui,la magie et l'imaginaire préhispanique, la littérature latino-américaine, les castors canadiens en Terre de Feu, entre autres; à Accra (Ghana), les discussions ont porté sur la relation profonde et difficile entre la côte occidentale de l'Afrique et le Brésil ; à Lama (Pérou), on a échangé sur l'apprentissage entre les communautés de Quechua Lamas, Naranjal et Anak Churuyaku - Valisho, travaux de préservation et de diffusion des savoirs indigènes dans les communautés traditionnelles de San Martin, dans la province d'Alto Amazonas, en recherchant l'incorporation de l'histoire de leurs propres communautés.

Au Brésil, le débat a porté sur l'écosystème fragile du cerrado dans la Chapada dos Guimarães, et à Cuiabá sur le thème de la monoculture et la problématique de l'extinction des espèces de la flore indigène a alors émergé. Enfin, à São Paulo on a échangé sur l'art et 
l'architecture des nations Terena et Krenak, ainsi que sur le projet “ La ville que nous voulons séminaires " publics ${ }^{12}$.

Ensuite, des « Journées d'Étude » ont été organisées lors la 32ème Biennale. Pour aborder le sujet L'Incertitude Vivante, 81 artistes de 33 pays ont été invités à développer des processus créatifs sur le thème de l'incertitude dans des directions différentes. L'ensemble des poétiques visuelles a présenté des thèmes complexes, mettant en lumière des conflits latents par le biais de différents dispositifs et perspectives poétiques. Brièvement, les commissaires présentent les œuvres de quatre façons: dans une première, il y a les ouvrages qui traitent directement de la nature et des processus biologiques; dans une seconde, il y a quelques œuvres qui cherchent des récits et des formes de savoir ; dans une troisième, il y a la production artistique qui postule de façon critique les structures politiques, économiques, et de la puissance de la représentation, et enfin, les œuvres qui suscitent l'imagination vers $\mathrm{d}^{\prime}$ autres modes de vie ${ }^{13}$.

Figure 1 : XXXII Biennale vidéo dans les villages



Je mets en évidence les œuvres d'Alia Farid, artiste koweïtien qui a produit une vidéo sur les ruines d'un projet d'architecture d'Oscar Niemeyer à Tripoli (Liban). Cet espace a déjà abrité des activités et des personnes diverses comme des réfugiés et des milices ou gardes de munitions de guerre. Le travail d'Alia Farid pose le problème de la dimension politique de ces ruines tout en ouvrant sur des recherches propres aux représentations du paysage du moderniste. Misheck Masamvu, un artiste qui vit à Harare (République du Zimbabwe) a produit beaucoup de toiles (huile sur toile) intitulées Spiritual Host et Midnight avec des formes abstraites, des parties d'êtres humains et des êtres surnaturels anciens, renvoyant sans doute à la situation postcoloniale comme un projet incomplet dans le pays. Lais Myrrha (São Paulo, Brésil) présente l'œuvre Deux poids, deux mesures (2016) composée par deux tours massives, mais de la même taille : l'une est constituée de matériaux de construction indigènes et l'autre de matériaux de construction de la modernité 
brésilienne. Par ces réalisations, elle cherche à articuler des outils et des savoirs. Grada Kilomba (Lisbonne, Portugal) dispose, quant à elle, dans cette biennale de deux projets The Desire Project (2015-2016) qui est une vidéo installation sur des récits silencieux où le seul élément visuel est le mot; l'autre projet est une performance, avec des projections sur son corps. Dans cette oeuvre, l'artiste utilise les méthodes de la tradition africaine pour raconter des histoires, il déclare d'ailleurs ceci : "Donc je fais un panorama pour parler de thèmes postcoloniaux à travers différentes histoires, de plusieurs contes et j'essaie de faire un lien entre l'un et l'autre. ${ }^{14 "}$. Ce qu'elle veut finalement dans son ouvrage, c'est décoloniser la pensée.

En ce sens, la 32ème Biennale de São Paulo propose une cartographie des artistes, des chercheurs et des gestionnaires du paysage en Amérique latine attentifs à la conscience écologique concernant les soi-disant catastrophes environnementales, en cherchant une pensée autonome qui rende compte des conditions de ce qu'on appelle «Sud Global ».

Pour cela, ils convoquent des chercheurs de renom tels que Boaventura dos Santos et s'appuient sur Viveiros de Castro en tant que représentant de cette pensée autonome ainsi que sur des artistes qui rétablissent des poétiques visuelles et sonores à partir de savoirs et pratiques traditionnelles oubliés par la culture appelée « Nord Global ».

La pensée de Boaventura de Souza Santos, décrite dans le catalogue de l'Exposition ('Incertitude entre la peur et l'espoir ${ }^{15}$ - a un fort impact sur la conception de cette Exposition d'Art ${ }^{16}$; dans son texte, il s'interroge sur l'époque que nous vivons et sur les incertitudes qui nous entourent .En 1995, cet auteur a proposé le concept d'« épistémologies du Sud», concept qui a donné lieu à de nombreux débats dans le milieu spécialisé, le menant d'ailleurs à écrire plusieurs autres articles et livres sur le sujet. Cependant, l'affirmation de ce travail, selon laquelle "Une épistémologie du Sud repose sur trois principes directeurs : apprendre qu' existe le Sud; apprendre à aller vers le Sud; apprendre à partir du Sud et avec le Sud» (SANTOS, 1995 : p. 508) mobilise le champ de l'art contemporain.

Pour l'auteur Maria Paula Meneses, qui organise l'œuvre «Épistémologies du Sud avec Boaventura dos Santos ", les réalités qui sont maintenant désignées comme postcoloniales ne peuvent pas avoir comme référence un modèle unique. La diversité de l'Amérique latine est distincte de celle qui se produit en Afrique ou dans des contextes européens. Et, au sein de chacun de ces macrocosmes, il y a une multitude de microcosmes, tous infiniment différents les uns des autres. Toutefois, si cette différence d'espace et de temps fait appel à la différence dans le Sud, l'expérience coloniale commune permet la création d'un Sud global, où la condition postcoloniale s'impose de plus en plus dans l'analyse et la caractérisation des conditions politiques spécifiques ${ }^{18}$.

Ce panorama artistique instauré par la 32ème Biennale de São Paulo a été conçu avec les savoirs et les pratiques d'un territoire dénommé «Sud Global »; ce concept, néanmoins, n'est pas configuré par des frontières nationales. De plus, l'ample matériel édité au cours des recherches a été réalisé à partir des pratiques collectives de la connaissance et des poétiques visuelles pour le développement d'une esthétique et d'une politique de la nature en crise.

Par ces affirmations, les artistes visuels cherchent à expliciter les conflits sociaux et politiques, l'approfondissement et l'expansion de la démocratie à travers l'activisme artistique dans le sens arendtien de la sphère publique ${ }^{19}$; loin des concepts qui lient la sphère publique comme un champ conceptuel homogène et validateur d'idées avec des vues apaisantes de la vie sociale qui forme des identités et des actions de citoyenneté. 
21 Au moment où l'art cherche sa connexion avec la vie, occupant la rue et s'approchant de communautés, nous sommes en présence d'un espace public érosif qui ne se propose pas d'examiner la question sociale, mais de mettre en évidence les multiples fractures entre la société et l'espace politique et urbain. Les collectifs d'art, les artistes plasticiens et les critiques d'art avaient défini l'espace public comme une arène d'activité politique, et cherchaient à redéfinir l'art public comme un art qui participe ou crée un espace politique. Cet activisme esthétique et social a trouvé une source dans la catégorie de "sphère publique ", qui est désormais librement utilisée pour désigner le domaine d'une interaction discursive autour de questions politiques, en désignant l'espace public urbain comme un espace de conflits ${ }^{17}$.

L'organisation de grandes et importantes expositions d'art contemporain, lors desquelles les commissaires font des propositions distinctes de celles de l'art produit dans les territoires du“ Nord Global », ou « Nord-Ouest », permet de donner de la visibilité à la production artistique des pays qu'on nomme «Sud Global». Celles-ci ont constitué une pratique artistique significative, ainsi que le prouve la proposition de la Documenta 14.

Ces thèmes se sont dégagés de l'Exposition de la 32ème Biennale avec une grande éloquence permettant à ces communautés et aux conflits de constituer une moisson de la sphère publique dans le sens arendtien ${ }^{18}$.

À propos de la notion de sphère publique chez Hannah Arendt, la professeure et critique d'histoire d'art Rosalyn Deutsche a abordé très clairement, dans l'article présenté à l'Exposition Arco à Madrid, en 2008, les notions de publique, d'art dans la sphère publique, $\mathrm{d}^{\prime}$ alterité et de politique. Pour Deutsche, la philosophe Hannah Arendt a défini la sphère publique, ou la communauté politique-démocratique, comme "l'espace d'apparition ", ou ce que la phénoménologie appelle "rendre visible» (DEUTSCHE,2009:175). En soulignant l'apparition, Arendt connecte la sphère publique - qu' elle a conçue à partir de la polis grecque - «à l espace de l'apparition, à la vision » ${ }^{19}$.

Dans sa célèbre phrase, Arendt a écrit :

«La polis (...) n'est pas la cité-Etat dans son espace physique ; c'est l'organisation des personnes à mesure qu' agir et paler apparaissent ensemble, et son véritable espace est parmi les gens qui vivent ensemble dans ce but, où qu'ils soient (...) est l'espace d'apparition dans le sens le plus ample du mot, soit l'espace où j'apparais aux autres à mesure que les autres m'apparaissent, où l'homme (...) fait son apparition explicitement » (ARENDT,2010, p. 198-199).

Dans ce sens, les collectifs artistiques ou les artistes visuels avaient travaillé individuellement sur des interventions dans l'espace public.La constitution de la sphère publique et la problématisation d'espace de la vision et de l'apparition dans les arts visuels, au sens large du mot dans la polis grecque, sera explicitement reprise dans la Documenta 14, en 2017, qui aura lieu simultanément à Kassel (Allemagne) et à Athènes (Grèce). Organisée par Marina Fokidis à Athènes sur le thème - Apprentissage avec Athènes -, l'exposition aura une signification symbolique dans le sens moderne de la démocratie. Dans cette édition, l'exposition d'art Documenta 14 se produira simultanément entre le soi-disant Nord-Sud sur la proposition du commissaire Adam Szymczk.

28 Marina Fokidis, directrice artistique de la Documenta 14 à Athènes, était à un Séminaire sur l'art contemporain et la réalité “, événement parallèle à la 32ème Biennale de São Paulo, où elle a fait une conférence sur les directives de l'Exposition à Athènes, dont le thème de la Documenta 14 sera " Apprentissage avec Athènes »; c'est dans cette édition, 
comme indiqué ci-dessus, dans son sens symbolique et, aussi, comme une métaphore de la fondation du sens moderne de démocratie avec la création d'une sphère publique. Elle considère la notion de constitution d'un « Sud global » comme un état d'esprit et qui n'est pas nécessairement associé à un terme géographique; "nous pensons le sud comme un espace où les gens se rencontrent pour imaginer la possibilité d'autres formes d'être dans le monde ». Une « petite sphère publique » (FOKIDIS, 2016 : p. 29) ${ }^{20}$.

\section{Le paysage dans l'œuvre de Carolina Caycedo} pur de l'épistémologie du Sud, qui pourraient, à notre avis, reconfigurer un large champ d'expériences poétiques aux pouvoirs différents, mis en place dans la 32ème Biennale d'Art de São Paulo, en niant toute possibilité de matrices discursives nostalgiques de formes de vie passées. Carolina Caycedo et Alia Farid ${ }^{22}$, parmi d'autres grands artistes de la 32ème Biennale, exposent des représentations du paysage qui cherchent la décolonisation des connaissances et des pratiques artistiques.

La pratique artistique de Carolina Caycedo, artiste colombienne née à Londres et qui vit actuellement entre La Jagua (Colombie) et Los Angeles (USA), exposée à la 32ème Biennale de São Paulo, part de la recherche de contextes sociaux et environnementaux liés aux projets de barrages hydroélectriques enrelation avec les peuples riverains. A partir de l'étude initiale, elle recherche les flux des eaux, l'assimilation, la résistance, la représentation, le contrôle, enfin les relations entre la nature et la culture; grâce à son engagement envers les communautés riveraines; elle apprend que, "je suis en train de comprendre un barrage comme structure qui contient un plan d'eau, mais qu'il y a aussi une relation avec le confinement et la répression des communautés qui coexistent avec la rivière ${ }^{23}$.

Dans son projet artistique appelé The People River - Be Dammed (2016), elle étudie l'impact socio-culturel de la construction de centrales hydroélectriques dans les Amériques, en utilisant le mot «dam» dans un double sens (barrage, étang) et «damn» (malédiction). Dans sa recherche récente, elle examine les dommages environnementaux et sociaux résultants de la construction de barrages et du contrôle des cours d'eau naturels. The People River - Be Dammed (2016) est le résultat d'un projet initié par l'artiste, en 2012, qui se compose de dossiers de recherche, $d^{\prime}$ études de terrain et d'activités auprès des communautés riveraines affectées par la privatisation de l'eau. 
34 Au Brésil, son travail a été potentialisé par le documentaire produit par le Mouvement des Atteints par des Barrages, MAB, dédié à la mémoire de Nilce de Souza Magalhães, une des sources d'inspiration de l'artiste Carolina Caycedo pour sa participation à la 32ème Biennale. $)^{24}$.

35 L'œuvre artistique de Carolina Caycedo dans la 32ème Biennale de São Paulo « Le peuple Xingu, Le peuple Doux » occupe plusieurs espaces physiques et narratifs. Elle est composée de différents dispositifs, parmi eux, un grand panneau résultant de l'installation de photographies satellite de l'Usina de Itaipu (sur la frontière entre le Brésil et le Paraguay, à Foz do Iguaçu, bassin hydrographique du Rio Paraná), de l'usine hydroélectrique de Belo Monte (bassin hydrographique de Rio Xingu, du bassin de l'Amazone, en particulier dans l'état du Pará et du barrage de Bento Rodrigues (ville de Mariana, Minas Gerais, Brésil, bassin hydrographique de Rio Doce).

Figure 2 : Carolina Caycedo, Le peuple Xingu, Le peuple Doux, de la série The People river -Be Dammed

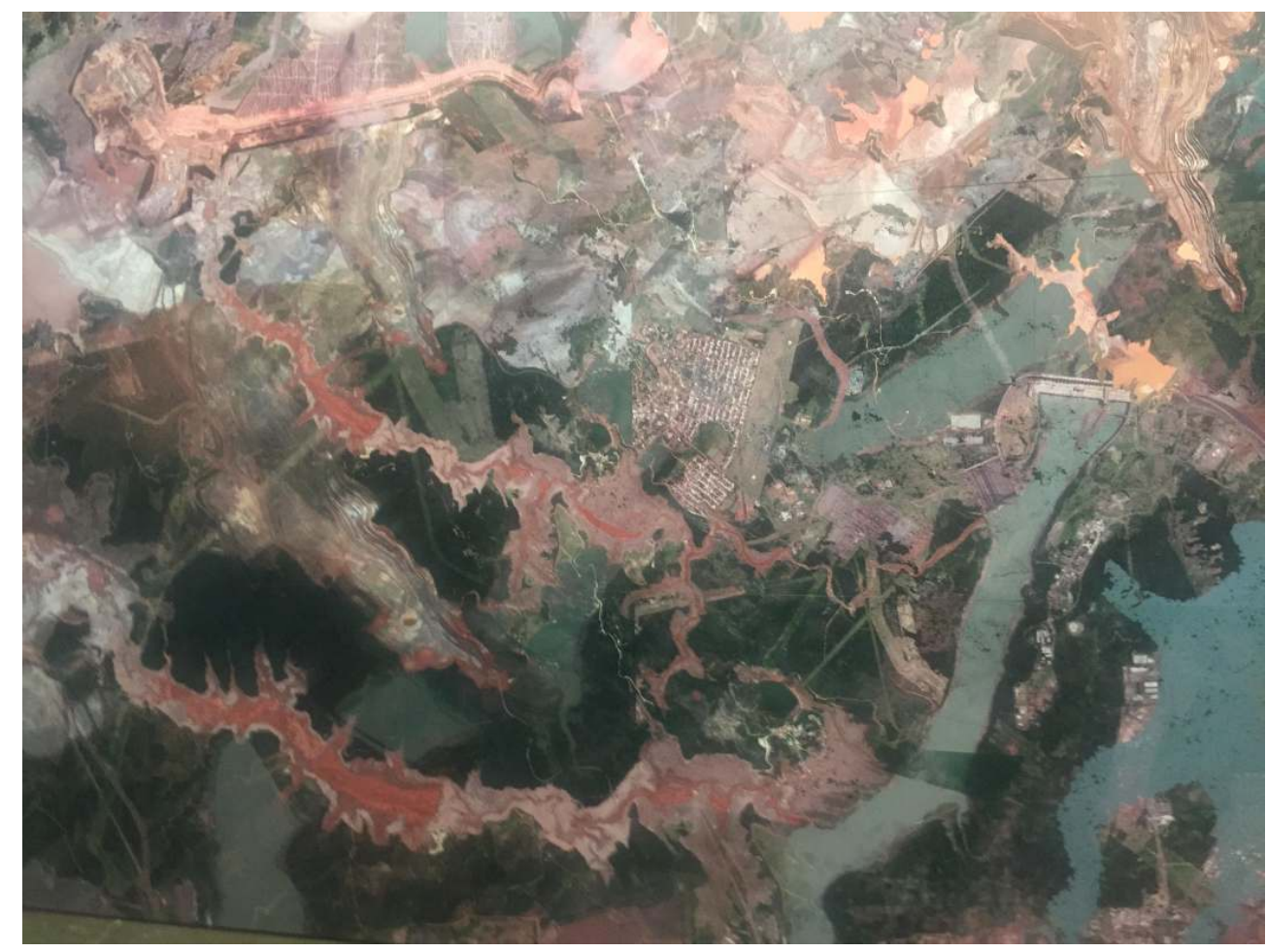

Ce panneau, fait par impression UV - ultraviolet - sur aluminium Dibond (Photo 2), représente le thème du paysage dans l'art contemporain ; ainsi que des dessins, avec des marqueurs et à l'encre sur papier Canson, qui racontent les récits des rivières Yuma (Colombie), Yaqui (Mexique), Elwha (USA), Watu (Brésil), également connues sous le nom de Rio Doce et le Rio Iguaçu (Brésil) ; ces rivières sont représentées et enregistrées comme des entités vivantes qui ont leurs propres histoires (photos 3 et 4).

Figure 3 : Carolina Caycedo, dessins 


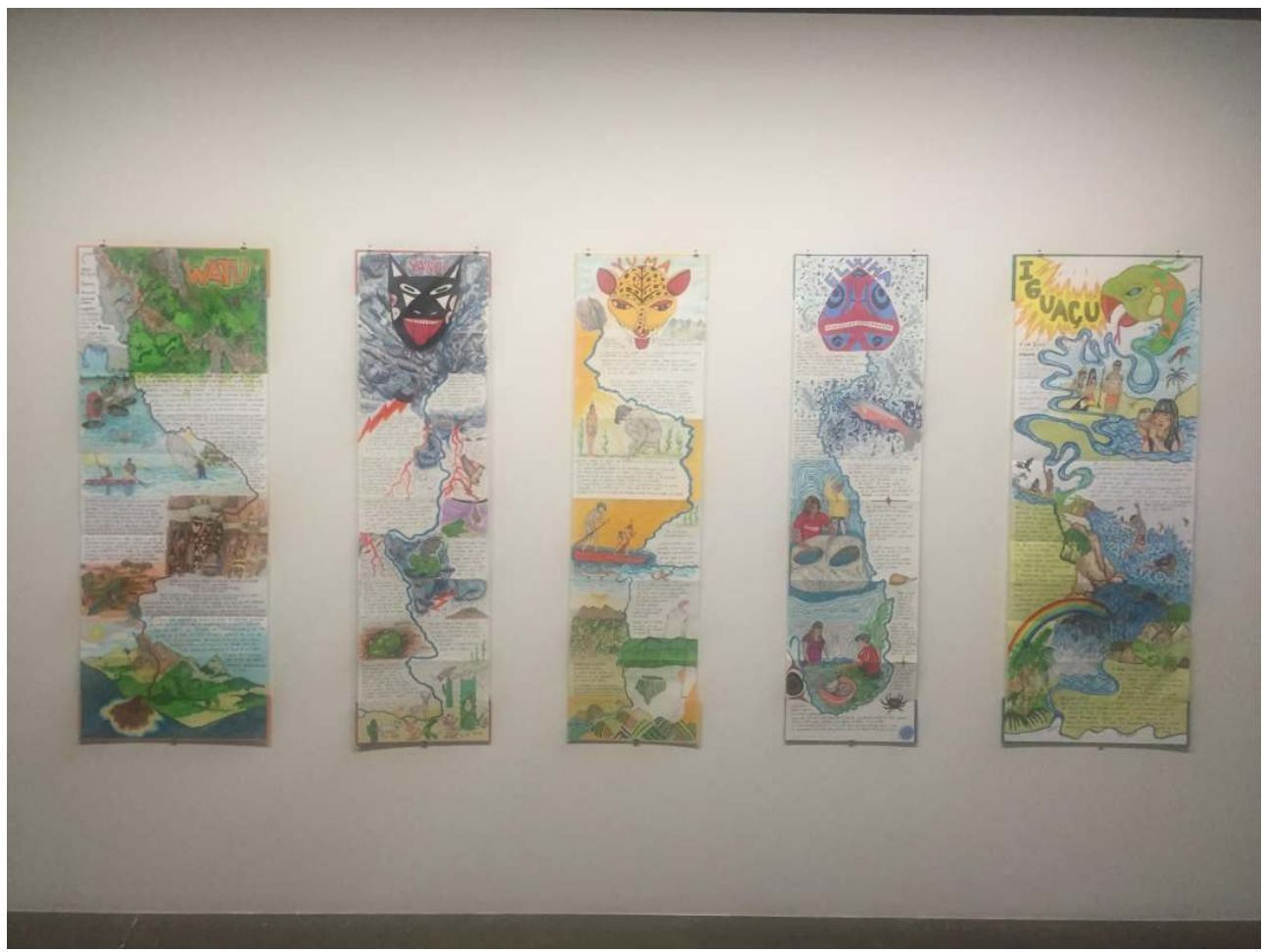

marqueurs et encre sur papier Canson

Figure 4 : Carolina Caycedo,-dessins

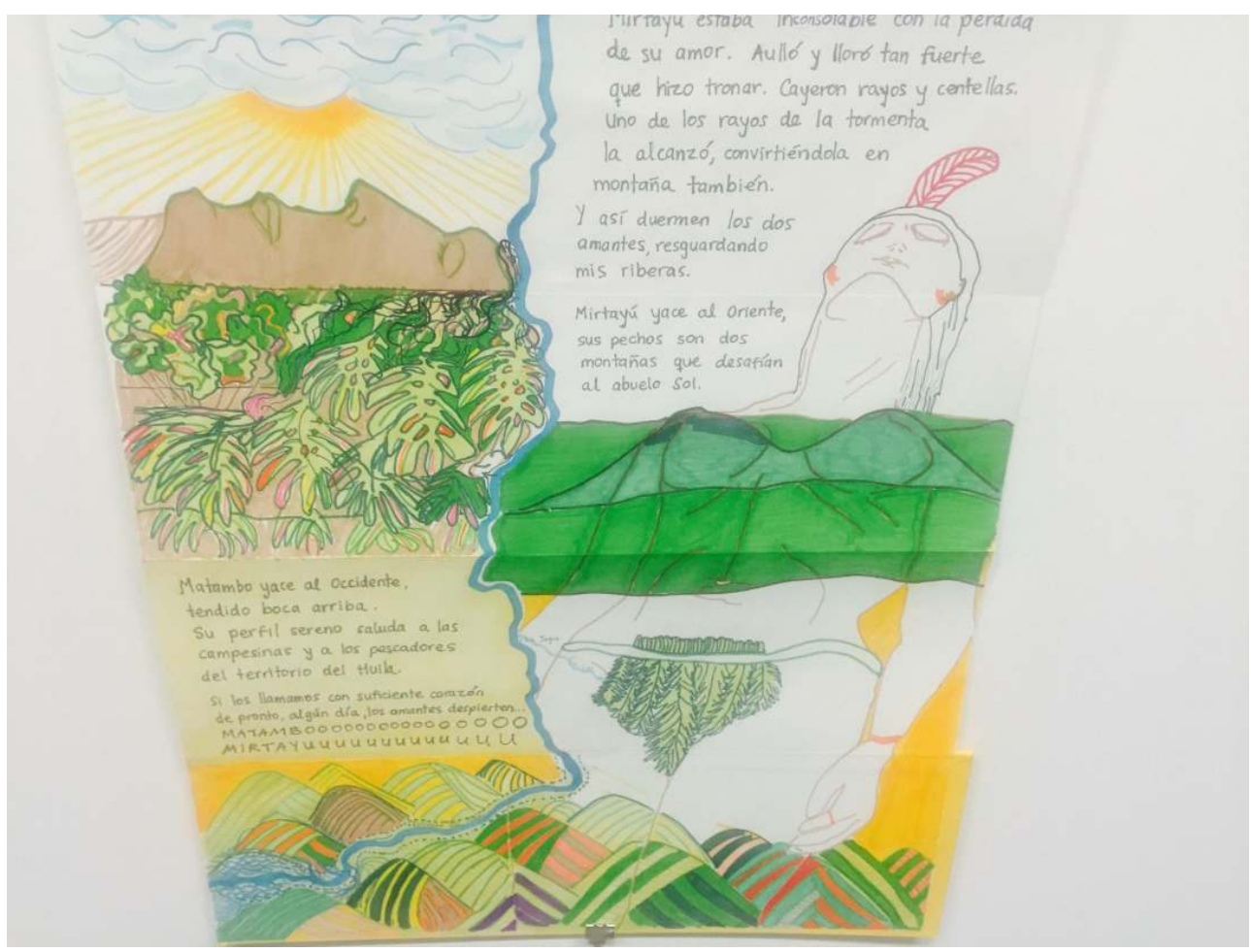

marqueurs et encre sur papier Canson

37 À la Biennale est présentée aussi une vidéo filmée par Caycedo dans la région de Bento Rodrigues, avant et après le plus grand accident environnemental de l'entreprise minière 
dans l'histoire du Brésil, qui résulte de la rupture du barrage de résidus d'un grand consortium minier international. L'artiste affirme que - "l'art est le meilleur outil pour comprendre les injustices et pour participer aux processus d'organisation et de lutte» (CAYCEDO,2016:1). Dans cette Biennale est également presentée une œuvre de cette artiste constituée par des éperviers de pêches (réseaux) contenant des matériaux recueillis lors de recherche de terrain et insérés dans les espaces entre les différents niveaux du Pavilhão de la Biennale (photos 5 et 6).

Figure 5 : Carolina Caycedo, Réseaux

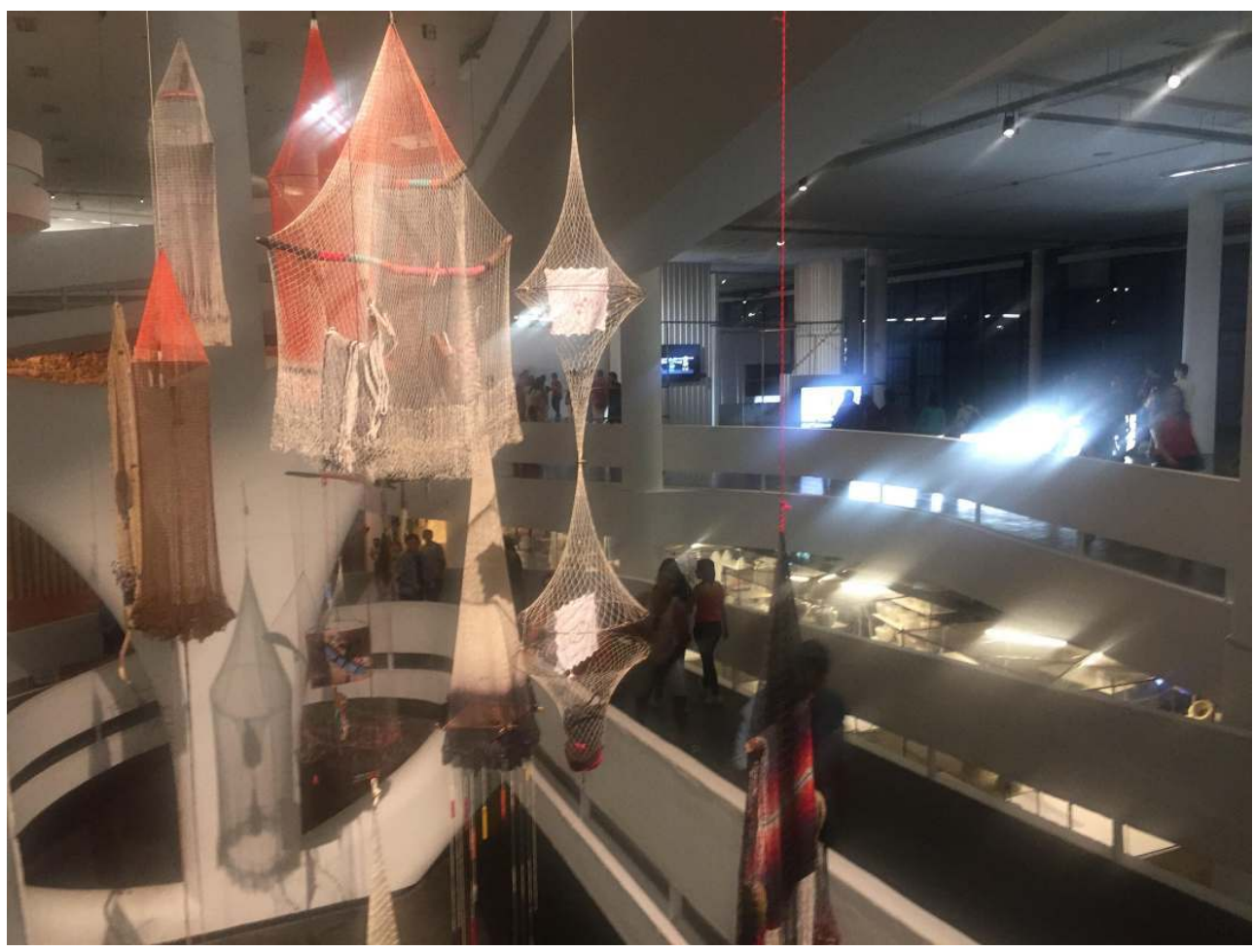

matériaux recueillis 
Figure 6 : Carolina Caycedo, Réseaux

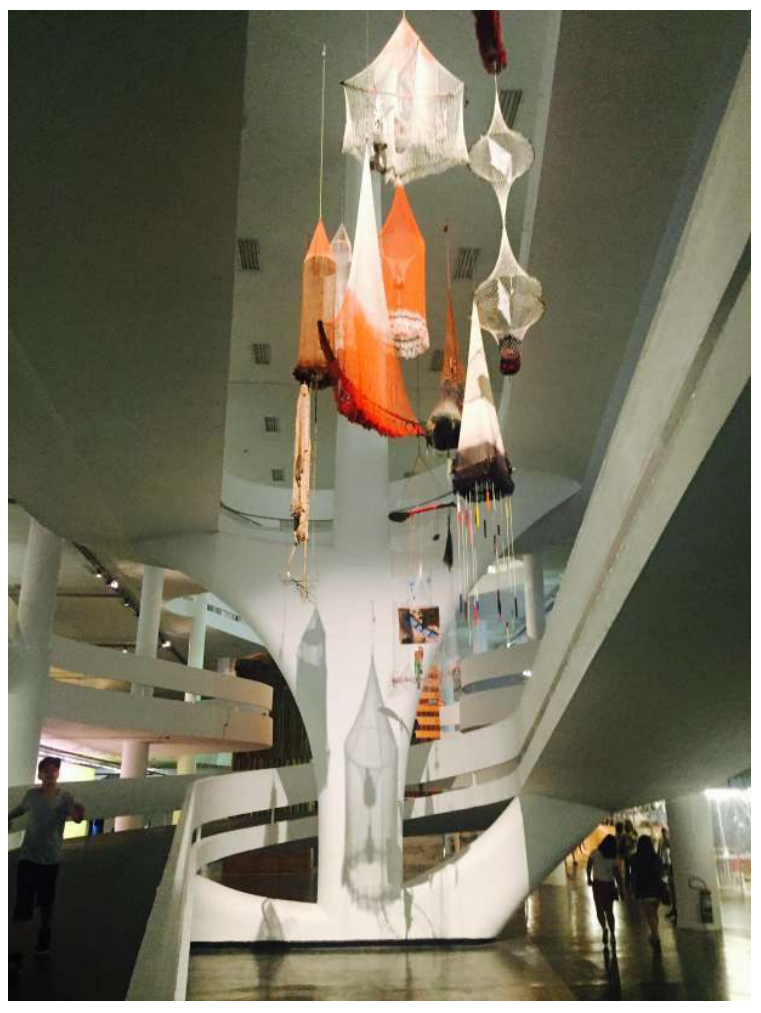

Sa pratique artistique, comme le souligne l'anthropologue Fabio Zuker, a une dimension collective. Dans cette pratique, les performances, les dessins, les photos et les vidéos ne sont pas simplement le résultat final, mais une partie du processus de recherche et d'action de l'artiste (ZUKER, 2016: p. 116). C'est dans cette perspective que nous comprenons la recherche et l'intervention poétique que développe Carolina Caycedo, depuis 2012, cette approche a d'ailleurs déjà entraîné le travail Geochoreographies Oritoguaz, | GEO-choreographie communitaire, dans l'endroit prévu pour la centrale hydroélectrique Oporapa, sur la rivière Yuma, à Huila, Colombie, nommée Rio Magdalena par les colons espagnols. Les Geochoréographíes sont chez Cayedo comme les gestes du quotidien qui se répètent et qui sont étroitement liés à une géographie, dans ce cas, les rivières: "lorsque cette géographie disparaît parce qu'elles sont inondées ou contaminées, les pêcheurs ne peuvent plus exercer ce geste qui est une connaissance incarnée, transmise depuis des générations ", ajoute-telle (MOLINA, $2016: 1)^{25}$.

Au Brésil, Caycedo a participé comme artiste invitée lors des "Journées d'Etude" du Programme de la 32ème Biennale de SP, qui onteu lieu dans les villes citées plus haut. L'artiste Caycedo a participé, essentiellement à Cuiabá (État de Mato Grosso, Brésil) à la Journée d'Etudes sur le domaine paysager du cerrado, un des biomes les plus fragiles et qui, comme porte d'entrée de l'Amazonie, forme aujourd'hui comme un espace de terres en monoculture où les savoirs traditionnels ont été oubliés. À São Paulo on a échangé sur l'art et l'architecture des nations Terena et Krenak.

Elle a également visité la région du Rio Doce sinistrée par une catastrophe écologique (Mariana, Minas Gerais), ce qui lui a permis de réaliser un essai vidéo présenté lors de cette Biennale. Auparavant, elle a fait des recherches dans le bassin du Rio Iguape (Cananeia, littoral sud de São Paulo, Brésil), où elle vivait avec les pêcheurs de Cananeia et 
l'ancien "quilombo" de Ivaporunduva"26, préservé en raison de son isolement, dans la vallée du Rio Iguape, littoral sud de l'État de São Paulo. En vivant avec les pêcheurs à Cananeia, elle a produit des travaux en utilisant des éperviers et des matériaux recueillis dans la région, travail artistique prestigieux présenté à la 32ème Biennale. Sur cette œuvre, Cayedo dit que "la Ribeira est une rivière qui n'est pas retenue et donc il y a des quilombos et des communautés de pêcheurs qui résultent des processus de résistance, processus autonomes de vivre avec l'eau et les poissons » (MOLINA, $2016: 1)^{27}$.

\section{Une approche aux paysages critiques ou paysages du sud}

L'analyse discursive dans ce texte sur l'oeuvre Le peuple Xingu, Le peuple Doux, de la série The People river-Be Dammed, de Carolina Caycedo dans la Biennale de São Paulo, propose de montrer que la construction des répresentations du paysaje qu' elle réalise rend compte d' une pensée autonome sur l'art contemporain dans ce qui est dénommé "sud global".

Les représentations visuelles qui rendent compte $d^{\prime}$ une pensée autonome sur l'art contemporain sont antérieures à cette Biennale de São Paulo Il faut, toutefois, rappeler que les propositions vers la construction d'une pensée autonome qui rendent compte des conditions réelles de ce qu'on appelle "Sud global», dans les arts visuels, ont des rapports avec diverses propositions depuis des décennies.

D'ailleurs, nous mettons en évidence le projet du critique d'art Mario Pedrosa (1900-1981), dans les années 1970, d'une Exposition au Musée d'art moderne de Rio de Janeiro), qui a réuni la collection de plusieurs institutions, notamment des œuvres et des objets d'art de différentes ethnies du Brésil. L'exposition s'appelle «Alegria de Viver, Alegria de Criar » (Joie de vivre, joie de créer) et, après l'incendie de MAM-RJ, le projet de création du Musée des origines devait rassembler plusieurs centres d'interêts : Museu do Índio - Musée de l'Indien, Museu de Arte Virgem - Musée d'Art Vierge ou Museu do Inconsciente - Musée de 1' Inconscient, Museu de Arte Moderna - Musée d'Art Moderne, Museu do Negro - Musée du Noir, et Museu de Artes Populares - Musée des Arts Populaire., Cette dernière proposition de Pedrosa résulte d'une attitude de désenchantement par l'art et par la dimension esthétique dans le monde, comme une possibilité de créer des bases puissante pour l'art contemporain ${ }^{28}$.

4 La "Mostra do Redescobrimento" (Exposition de la Redécouverte - Fundation Biennale de São Paulo, 2000) a été conçue à partir de la proposition du critique Mário Pedrosa, pour la création du Musée des Origines, en 1978, selon le commissaire de la Exposition de la Redécouverte - Nelson Aguilar. Plus récemment, certains projets artistiques des commissaires, réunis autour de la recherche de l'intégration de la question amérindienne dans l'art contemporain, comme La Chute du Ciel ( A Queda do Céu , commissaire Moacir dos Anjos, Paço das Artes, São Paulo, 2015); Histoires Mestissées (Histórias Mestiças ,commissaires Adriano Pedrosa et Lilia Schwarcz, Instituto Tomie Otake, São Paulo, 2015) et 34ème Panorama de l'Art Brésilienne Contemporaine ( XXXIV Panorama de Arte Brasileira Contemporânea, commissaires Aracy Amaral et Paulo Miyada, Museu de Arte Moderna, São Paulo, 2015).

Dans l'exposition Histoires Mestissées, le commissaire a fait remarquer dans le Catalogue que «l'imposition de la notion d'objet d'art correspond à une "européanisation du monde $»^{29}$, 
remise en question depuis les études post-coloniales"; question qui a guidé la conception conservatrice et expographique de l'exposition. La visibilité de la production artistique de ce qu'on appelle « Sud global » et l'importance des savoirs traditionnels initient la 32ème Biennale de São Paulo. Dans son essence, la notion d'Incertitude Vivante en tant que poétiques visuelles unissant des récits comme laréflexion et la pratique artistique, loin des conceptions muséologiques ethnographiques; mais portée vers la recherche «d'autres façons de penser » les communautés étudiées lors des «Journées d'Études » et les incertitudes que posent à l'avenir l'émergence des questions environnementales.

D'autre part, nous citerons quelques oeuvres d'artistes visuels qui ont fait des représentations picturales du paysage dans l'art contemporain. La peinture de paysages élaborée par des peintres voyageurs comme Albert Eckout (1610-1666) ou Frans Post (1612-1680), entre autres, a longtemps été revisitée dans l'art contemporain, révélant la construction culturelle d'un imaginaire nuancé par les processus de la colonisation européenne du continent latino-américain; ainsi que la peinture du paysage dans les oeuvres des artistes voyageurs du XIXe siècle tels qu'Alexander von Humboldt et Johann Moriz Rugendas, qui ont contribué à créer un imaginaire idyllique et qui ont influencé la formation des identités nationales.

Cette construction culturelle avait été révélée, à l'époque contemporaine, par l'historiographie critique ${ }^{30}$ et par des œuvres visuelles contemporaines qui soulignaient la pensée colonisatrice ou le regard européen dans les représentations de la nature, notamment la peinture du paysage des artistes voyageurs. Une artiste des plus contondantes dans sa poétique critique de la pensée colonisatrice dans les représentations de la nature par la peinture des artistes voyageurs, c'est Adriana Varejão. L'artiste, dans son œuvre Paysages (1995) s'approprie une œuvre de l'artiste voyageur le Comte de Clarac (1777-1847) dont l'aquarelle intitulée Forêt Vierge du Brésil «Floresta Virgem do Brasil» (1819), en y ouvrant une fracture dans l'image avec des couches d'encre couleur de sang. Dans l'œuvre Viandre à la Mode Frans Post (1996) (Carne à Moda de Frans Post) ${ }^{31}$, Adriana Varejão, par le biais de sa poétique visuelle, révèle une construction culturelle d'un pays idyllique conçue par l'artiste voyageur Frans Post, créant un nouveau récit, ou selon les mots du commissaire Paulo Herkenhoff, l'artiste Adriana Varejão veut mettre en évidence une histoire tragique qui favorise une élucidation dialectique du présent $t^{32}$.

Dans Carte de "Lopo Homme", (Mapa de Lopo Homem), huile sur bois et suture (1992), l'artiste prend comme point de départ un ensemble de dix cartes nautiques de 1519 faisant partie de l'Atlas nautique du monde, nommé Atlas Miller, dans laquelle l'artiste reprend le thème de la cartographie. A titre d'exemple le travail de Joaquín Torres-García (1943) «L’Amérique Inversée » qui renverse les coordonnées géographiques de la carte de l'Amérique du Sud, en mettant le Sud à la place du Nord ; l'artiste Varejão fait une grande déchirure dans cette carte nautique et bouleverse la géographie du raccordement des continents et des dénominations. Les références utilisées par l'artiste dans ce travail montrent son intérêt dans le développement de nouvelles narratives sur le thème de la domination culturelle européenne.

Pour reprendre le travail de Carolina Caycedo pour la 32ème Biennale, nous voyons le montage des œuvres et des divers dispositifs artistiques, dans lequel l'image est le temps. L'œuvre Le peuple Xingu, Le peuple Doux, composée par le chevauchement d'images bidimensionnelles et sans aucune profondeur optique, realisées par satellites, des images des forêts du domaine paysager de l'Amazonie et des images de la forêt Atlantique 
coupées par des barrages et des centrales hydroélectriques. Ce paysage, loin de révéler des sentiments du sublime qui va vers l'incalculable, qui va au-delà de l'homme et de toutes mesures dictées par les sens, à l'infini, révèle son revers - dans le paysage Le peuple Xingu, Le peuple Doux - la nature ne menace pas, n'est pas terrifiante par son immensité. Dans les paysages, ce sont les interventions humaines signalées par l'eau de couleur d'argile des rivières qui coupent les forêts, qui menacent la nature et l'homme lui-même. Ces paysages, loin de montrer l'utopie de la modernité, portent le sens le plus éloquent de la dystopie du projet moderne, l'inexorabilité de la finitude des ressources de la nature. Devant les ruines et la saturation de matériaux polluants qui coulent dans les rivières; nous n'avons pas dans cette figure d'image satellite de différents types de temporalités et de mémoire, ce sont des paysages sans mémoire.

51 Ces différentes temporalités et mémoire sont reproduites dans les dessins sur papier Canson, qui racontent les récits des rivières Yuma (Colombie), Yaqui (Mexique), Elwha (USA), Watu, aussi appelée Rio Doce et Iguaçu (Brésil) ; ces rivières sont représentées et enregistrées comme des entités vivantes qui ont leurs propres histoires dans différentes temporalités.Ces dessins rappellent le travail des bordeuses, dont parle Caycedo dans un entretien avec Louise Lobler, activiste du MAB- Moviment des Victimes des Barrages, sur le Projet $\mathrm{d}$ 'Arpilheiras. Les arpilleras sont un type d'emballage du jute, où on conserve des produits alimentaires : les sacs de jute. Au début du siècle dernier, au nord du Chili et en Bolivie, les femmes brodaient sur ces sacs, des scènes de leur vie quotidienne. Dans ces temps là, à la campagne, les paysans n'avaient pas accès à une caméra, mais il s'agissait d'un moyen de faire de constituer um témoignage, comme une photographie : broder un succès familial. Et les femmes construisaient leur histoire à travérs des images (CAYCEDO, 2016 : p. 1) ) $^{33}$.

De toute façon, l'ensemble des œuvres dans différents médias et dispositifs assemblés, dans le sens inscrit par Georges Didi-Huberman, de montage et remontage des œuvres d'art, et présentés par Carolina Caycedo, met en évidence les paysages critiques et leurs inscriptions et leurs représentations par des paradoxes et des conflits. Ainsi que l'a souligné le philosophe et historien Georges Didi-Huberman, «On n'expose poétiquement, visuellement, musicalement ou philosophiquement - la politique qu'à montrer les conflits, les paradoxes, les chocs réciproques dont toute histoire est tissée. C'est pourquoi le montage apparait comme la procédure par excellence de cette exposition (......)»(DIDI-HUBERMAN, 2007: p. 1) ${ }^{34}$.

\section{BIBLIOGRAPHIE}

ARENDT Hannah, A Condição Humana, Rio de Janeiro, Editora Forense Universitária, 2010.

BROWNLEE Peter John de, PICCOLI Valeria et UHLYARIK Georgiana Uhlyarik (édit.), Paisagem nas Américas : pinturas da Terra do Fogo ao Ártico, São Paulo, Édit. Terra Foundation, Pinacoteca do Estado de São Paulo, Art Gallery de Ontario, 2016.

CASTRO Eduardo Viveiros, DANOWSKI Déborah, Há um mundo por vir ? Ensaio sobre medos e fins, Florianópolis, Editora Instituto Socioambiental e Cultura e Bárbarie Editora, 2014. 
DANTo Arthur C., A Transfiguração do Lugar Comum : uma filosofia da arte, São Paulo, Éditeur Cosac Naify, 2005.

PEDROSA Adriano, Adriana Varejão : histórias às margens, São Paulo, Museu de Arte Moderna de São Paulo, 2013.

SANTos Boaventura de Sousa ; MENEZES Maria Paula (édit), Epistemologias do Sul, São Paulo, Editora Cortez, 2010.

voltz Jochen, REBouçAS Julia (édit.), Catálogo da $32^{\underline{a}}$ Bienal de São Paulo - Incerteza Viva, São Paulo, Fundação Bienal de São Paulo, 2016.

\section{NOTES}

1. VOLTZ, Jochen, REBOUÇAS, Julia (édit.). (2016). Catálogo da 32ª Bienal de São Paulo - Incerteza Viva. São Paulo : Fundação Bienal de São Paulo (Brésil). ARENDTH, 0-81. Editora Acrobá60-81, Editora Medo e a Esperan aos entulhos, a saturaositivos artina obra Floresta Virgem do B

2. Commissaires de la 32ème Biennale de São Paulo : Jochen Volz, Gabi Ngcobo, Julia Rebouças, Lars Bang Larsen et Sofía Olascoaga Julia Rebouças

3. SANTOS, B. S. (2008). A filosofia, a douta ignorância e a aposta de Pascal, Revista Crítica de Ciências Sociais, Coimbra (Portugal) ,80, pp. 11- 43 [DOI : 10.4000/rc cs .681).

4. SANTOS, Boaventura de Sousa. A Incerteza entre o Medo e a Esperança, dans : VOLTZ, Jochen, REBOUÇAS, Julia (édit,). Catálogo da $32^{\underline{a}}$ Bienal de São Paulo - Incerteza Viva. São Paulo : Fundação Bienal de São Paulo, 2016. ARENDTH, 0-81. Editora Acrobá60-81, Editora Medo e a Esperan aos entulhos, a saturaositivos artina obra Floresta Virgem do B

5. Voir en plus MARCONDES, Maria José de Azevedo. Prefácio : Natureza e Artifício. Dans : TERRA, Carlos ; ANDRADE, Rubens (édit.). Paisagens Culturais 4, Rio de Janeiro (Brésil) : Rio Books ; EBA UFRJ, 2014.

6. ROSSET, Clément. (1989). O naturalismo conservador ou a mística da falsificação, dans : A AntiNatureza : elementos para uma filosofia trágica. Rio de Janeiro : Édit. Espaço e Tempo, pp. 285-286

7. Voir en plus chez COHN, Danièle. (2012) Anselm Kiefer Ateliers. Éditions du Regard: Paris (France).

COELHO, Teixeira; MOLINO, Denis. (2010) Catálogo da Exposição. Romantismo: a arte do entusiasmo. São Paulo (Brésil) : Museu de Arte de São Paulo - MASP.

8. COHN, D. (2007). Compreender é julgar. Entrevista de Danièle Cohn a Glória Ferreira, Cezar Bartholomeu. Revista Arte \& Ensaios, Rio de Janeiro (Brasil), ano V, n 15, pp. 132-141.Disponible dans [ http://www.ppgav.eba.ufrj.br/publicacao/arte-ensaios-15]

9. CASTRO, Eduardo Viveiros. Déborah Danowski. (2014). Há um mundo por vir ? Ensaio sobre medos e fins. Editora Instituto Socioambiental e Cultura e Bárbarie et Editora Florianópolis (Brésil). Les auteurs sont cités dans l'article des conservateurs de l'Exposition avec la théorie de perspectivisme amérindien

10. Commissaires de la 32ème Biennale de São Paulo : Jochen Volz, Gabi Ngcobo, Júlia Rebouças, Lars Bang Larsen et Sofía Olascoaga.

11. Point de vue épistémologique de la connaissance pour la compréhension de la culture des natifs amérindiens, consolidée par l'anthropologue Eduardo Viveiros de Castro avec la crise de la pensée structuraliste. Voir en plus chez : VIVEIROS DE CASTRO, E. (1996). Os pronomes cosmológicos e o perspectivismo ameríndio. Mana, Rio de Janeiro (Brésil) v. 2, n. 2, oct, pp. 115-144

12. Source: VOLTZ, Jochen, REBOUÇAS, Julia (édit.) (2016). Catálogo da $32^{\underline{a}}$ Bienal de São Paulo Incerteza Viva. Fundação Bienal de São Paulo, São Paulo (Brésil).

13. Voir www.32bienal.org.br/pt/exhibition/ 
14. ROLNIK, S. (2016) O conhecimento e a colonização. Entrevista de Suely Rolnik com a artista Grada Kilomba, Revista Arte ! Brasileiros, São Paulo (Brésil), ano V, n 36, Brasileiros Editora, pp. 40-46.

15. SANTOS, Boaventura de Souza. A Incerteza entre o Medo e a Esperança, In VOLTZ, Jochen, REBOUÇAS, Julia (édit.) Catálogo da $32^{\underline{a}}$ Bienal de São Paulo - Incerteza Viva. Fundação Bienal de São Paulo, São Paulo,2016

16. SANTOS, Boaventura de Sousa. (1995) Toward a New Common Sense : Law, Science and Politics the Paradigmatic Transition. Routledge Publishing, New York.

${ }^{18}$ MENEZES, M. P (2008). Epistemologias do Sul, Revista Crítica de Ciências Sociais, 80, pp. 5-10.

${ }^{19}$ Voir en plus chez « DEUTSCHE, R.( 2009) . A Arte de ser testemunha em tempos de Guerra. Revista Concinitas, ano 10, volume 2, $\mathrm{n}^{\circ} 15, \mathrm{pp} .175-183 . »$

17. MARCONDES, Maria José de Azevedo. Arte Pública e Forma Urbana : o fetiche do espaço público. In : SEMINÁRIO ARTE E CIDADE, 2, Salvador, 2008. Salvador : FAU UFBA, 2008.

18. ARENDT, Hannah. (2010) . Condição Humana. Rio de Janeiro (Brésil) : Forense Universitária, 11.ed. Dans cet ouvrage, l'auteur développe le concept de la sphère publique.

19. DEUTSCHE, R. (2009) . "A Arte de ser testemunha em tempos de Guerra”. Revista Concinitas, ano 10 , volume $2, \mathrm{n}^{\circ} 15, \mathrm{pp} .175-183$.

20. MOCELLIN, V. 9 (2016). Um Sul sem Norte.Entrevista com Marina Fokids e Solange Farkas, Revista Arte ! Brasileiros, São Paulo (Brésil) ano V, n 36, , Brasileiros Editora, pp. 28-32.

Voir em plus chez https://www.deutschland.de/pt/topic/cultura/artes-arquitetura/ experimental-documenta-14

21. Voir en plus MARCONDES, Maria José de Azevedo. Prefácio : Natureza e Artifício. In : TERRA, Carlos ; ANDRADE, Rubens (édit. ) . Paisagens 4, Rio de Janeiro (Brésil) : Rio Books ; EBA UFRJ, 2014. 22. Voir en plus chez « Alia Farid - Ma 'arad Trablous », VOLTZ, Jochen, REBOUÇAS, Julia (édit.) (2016) Catálogo da $32^{\underline{a}}$ Bienal de São Paulo - Incerteza Viva. Fundação Bienal de São Paulo, São Paulo, pp. 87-89.

23. Voir en plus chez « Carolina Caycedo visita Mariana ao criar obra para a Bienal ». IN : Jornal do Commercio, Voir en http://jconline.ne10.uol.com.br/canal/cultura/artes-plasticas/ noticia/2016/06/15/carolina-caycedo-visita-mariana-ao-criar-obra-para-bienal-de-

sp-240264.php Consulté le 13 septembre 2016.

24. Connue comme Nicinha, la militante du MAB a été retrouvée et noyée dans le lac du barrage de l'Usine hydroélectrique Jirau, à Porto Velho (État de Roraima, Brésil). Voir en plus chez .Arte \& EnsaiosDocumentário do MAB inspira Carolina Caycedo, Disponible sur http:// www.32bienal.org.br/pt/collaboration/o/2715 Consulté le 25 septembre 2016.

25. MOLINA,C. L'écologie apparaît à différents niveaux à la 32e Biennale de São Paulo - Jornal Estado de São Paulo. Dans http://cultura.estadao.com.br/noticias/artes,a-ecologia-aparece-emdiferentes-niveis-na-32-bienal Consulté le 15 juin 2016.

26. Quilombo : lieu où s'établissemént des esclaves fugitifs

27. MOLINA, Camila. L'écologie apparaît à différents niveaux à la 32e Biennale de São Paulo Jornal Estado de São Paulo.

28. Voir en plus chez SANT'ANA, S. P. (2009) A crítica de arte brasileira: Mario Pedrosa, as décadas de 1950 et 2000 em discussão. Revista Poiesis, Rio de Janeiro (Brésil). Année X, n 14, pp. 17-33, Disponible sur http://www.poiesis.uff.br/PDF/poiesis14/Poiesis_14

ARANTES, Otília B. Fiori. (2004). Mario Pedrosa : itinerário crítico, Editora CosacNaify, São Paulo (Brésil), p. 168.

29. PEDROSA, Adriano, SCHWARCZ.L. (2015). Catálogo Histórias Mestiças, Editora Cobogó, São Paulo.

30. Une excellente contribution c'est le catalogue de l'exposition «Picturing the Americas. Landscape painting from Tierra del Fuego to the Artics». BROWNLEE, Peter John de, PICCOLI, Valeria et UHLYARIK, Georgiana Uhlyarik (édit.). (2016) . Paisagem nas Américas : pinturas da Terra do Fogo 
ao Ártico, Terra Foundation, Pinacoteca do Estado de São Paulo, Art Gallery de Ontario, São Paulo (Brésil)

31. Voir en plus chez PEDROSA, Adriano. Adriana Varejão : histórias às margens (curadoria e texto), Museu de Arte Moderna de São Paulo, São Paulo, 2013.

32. HERKENHOFF, Paulo. Glória, o grande caldo, Dans : Adriana Varejão. Takano Editora Gráfica, São Paulo, 2001.

33. Carolina Caycedo: Conversa sobre barragens com Louise Lobler (MAB). Dans: http:// www.32bienal.org.br/pt/soundfield/o/2856/ Consulté le 24 septembre 2016

34. DIDI-HUBERMAN, Georges .(2007) Remontée, remontage (du temps), dans : Revue Étincelle. IRCAM - Centre Pompidou, Paris. Disponible dans http://etincelle.ircam.fr/730.10.html Consulté le 30 juin 2016.

\section{RÉSUMÉS}

Cet article effectue une réflexion sur le travail Le peuple Xingu, Le peuple Doux, Doux, de la série The People river - Be Dammed, de l'artiste visuelle Carolina Caycedo, œuvre produite pour la 32ème Biennale de São Paulo, et analyse, à partir du travail artistique de Caycedo, le thème proposé par les commissaires de cette Biennale. Cette exposition dont le titre «L'incertitude vivante » a été structuré par une pensée épistémologique fondée sur le concept $d$ ' "épistémologies du sud", entendu comme la récupération de pratiques et de savoirs de populations. Cette conception imprègne d'importantes expositions d'art contemporain, produisant un impact sur les représentations du paysage dans l'art latino-américain de l'actualité.

Este artigo propõe uma reflexão sobre o trabalho A Gente Xingu, A Gente Doce, da série The People River - Be Dammed, da artista visual Carolina Caycedo, obra produzida para a $32^{a}$ Bienal de São Paulo e, analisa a partir do trabalho artístico de Caycedo o tema proposto pelos curadores desta Bienal. Esta Exposição denominada "Incerteza Viva" foi estruturada por um pensamento epistemológico fundado no conceito de "epistemologias do sul", entendido como a recuperação de práticas e saberes de populações tradicionais. Essa concepção tem permeado importantes mostras de arte contemporânea com impactos nas representações da paisagem na arte latinoamericana na atualidade.

\section{INDEX}

Palavras-chave : paisagem, arte contemporânea, representações, comunidades, saberes, descolonização

Mots-clés : paysage, art contemporain, représentations, art, communautés, savoirs, décolonisation 


\section{AUTEUR}

\section{MARIA JOSÉ DE AZEVEDO MARCONDES}

Professeure Docteure de l'Institut des Arts, Université d'Etat de Campinas, Brésil. Architecte et professeure docteure dans les cours de premier cycle en Architecture et Urbanisme, en Arts Visuels et au Programme du Doctorat en Arts Visuels, de l'Institut des Arts, Université d'Etat de Campinas, Brésil.

mmarcondes33[at]gmail.com 